\title{
AERODYNAMIC AND AEROELASTIC INSIGHTS USING EIGENANALYSIS
}

\author{
Jennifer Heeg * \\ NASA Langley Research Center \\ Hampton, Virginia \\ Earl H. Dowell ${ }^{\S}$ \\ Duke University \\ Durham, North Carolina
}

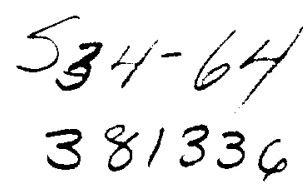

\begin{abstract}
This paper presents novel analytical results for eigenvalues and eigevectors produced using discrete time aerodynamic and aeroelastic models. An unsteady, incompressible vortex lattice aerodynamic model is formulated in discrete time; the importance of several modeling parameters is examined. A detailed study is made of the behavior of the aerodynamic eigenvalues both in discrete and continuous time. The aerodynamic model is then incorporated into aeroelastic equations of motion. Eigenanalyses of the coupled equations produce stability results and modal characteristics which are valid for critical and non-critical velocities. Insight into the modeling and physics associated with aeroelastic system behavior is gained by examining both the eigenvalues and the eigenvectors. Potential pitfalls in discrete time model construction and analysis are examined.
\end{abstract}

\section{INTRODUCTION}

A standard procedure for solving a structural dynamic problem is to employ eigenanalysis to calculate the structural dynamic eigenvalues and eigenmodes. Recently, this procedure has been extended to unsteady aerodynamics, and to coupled aeroelastic equations ${ }^{1,2}$.

Because applying eigenmode analysis to aerodynamic and aeroelastic systems is fairly new, there are many modeling issues that may not be familiar to the analyst. The intent of this paper is to discuss several of the many issues which are

N Aerospace Eng., Aeroelasticity Branch

$\S$ Professor, Dept of Mechanical Engr and Matl Sci; Dean, School of Engineering; Fellow AlAA

Copyright (C) 1999 by the American Institute of Aeronautics and Astronautics, Inc. No copyright is asserted in the United States under Title 17, U.S. Code. The U.S. Government has a royaltyfree license to exercise all rights under the copyright claimed herein for Governmental Purposes. All other rights are reserved by the copyright owner. associated with these new aeroelastic techniques. To accomplish this, we analyze relatively simple fluid dynamics and aeroelastic models using discrete time techniques and eigenmode analysis.

In computational fluid dynamics, CFD, there are two approximations that are typically employed. One is the construction of a computational grid, which determines the limits of spatial resolution of the computational model. The second is the approximation of an infinite fluid domain by a finite domain. It is a principal purpose of the present discussion to demonstrate that the computational grid not only determines the spatial resolution obtainable by the CFD model, but also the frequency or temporal resolution that can be obtained. Also, as will be shown, the finiteness of the computational domain determines the resolution of the eigenvalue distribution for a CFD model. Both of these observations have important ramifications for assessing the CFD model and its ability to provide an adequate approximation to the original fluid model on which it is founded. To these ends, a finite-wake, time-domain, discretized vortex lattice aerodynamic model has been utilized.

This paper presents results of aerodynamic parametric variations. A detailed discussion of the trends produced by these systematic variations will be presented. The discussion includes the effects on both the discrete- and continuous-time eigenvalues. These studies give new insights into aerodynamic modeling in the discrete time domain including how one may construct reduced order aerodynamic models.

The aerodynamic model was also combined with timedomain discretized structural dynamic equations to examine the aeroelastic behavior of a typical section. Aeroelastic analyses are also discussed in terms of eigenanalysis results. Aeroelastic stability analyses generally focus on the migration of the eigenvalues as a function of the velocity or other flow parameter. Indeed, much flutter analysis in practice today uses at best only an approximation to the true aeroelastic eigenvalues ${ }^{3-7}$. Here, the true eigenvalues are found for all aeroelastic 
modes without iteration. Also in this study, the characteristics of the eigenvectors are examined as the aeroelastic system becomes unstable.

\section{Aerodynamic Modeling \\ AERODYNAMIC STUDIES}

A Vortex Lattice solution to Laplace's equation for incompressible two-dimensional flow is utilized in this study. We consider the flow over an airfoil with a certain number of vortex elements on the airfoil and in the wake. The airfoil is modeled as a 2dimensional flat plate. The airfoil and the wake are divided into segments, referred to as aerodynamic elements. Vortex lattice aerodynamics are generated by placing vortices of strengths to be determined at points on the airfoil and in the wake. Collocation or control points, usually located aft of the vortex locations, are points where the boundary conditions must be satisfied. Typical placement is for the vortices to be located at the $1 / 4$-chord points of the aerodynamic elements. The collocation points are typically placed at the $3 / 4$-chord locations of the elements.

The governing equations are presented by Hall in reference 1; they are briefly summarized here. There are 3 basic relationships, detailed in the following paragraph, which are combined to form the matrix equation

$$
[A]\{\Gamma\}^{n+1}+[B]\{\Gamma\}=\{w\}^{n+1} \quad(\text { Eqn.1) }
$$

where $n$ and $n+1$ denote the current and the next discrete time sample. $\Gamma$ is a vector of vorticities and $w$ is a vector of downwashes at each of the collocation points. The number of elements on the wing is denoted $M$, while the total number of elements is denoted $\mathrm{N}$.

Three basic relationships determine the contents of the $\mathrm{A}$ and $\mathrm{B}$ matrices seen in Equation 1. These represent $\mathrm{N}$ equations with $\mathrm{N}$ variables. The first of the three basic relationships equates the velocity induced by the discrete vortices at the collocation points to the downwash caused by the airfoil's motion. This relationship accounts for $M$ rows within equation 1. Applying Kelvin's theorem generates a second basic relationship utilized in deriving equation 1. Quoting Hall, "unsteady vorticity is shed into the wake; its strength is proportional to the time rate of change of circulation about the airfoil. ... The time step is taken to be equal to the time it takes the vorticity to convect from one vortex station to the next." This relationship accounts for the $(M+1)$ row of equation 1 . Once the vorticity has been shed into the wake, it is convected in the wake at the freestream velocity. This is the third basic relationship and appears in equation $I$ in rows $(\mathrm{M}+2)$ through $(\mathrm{N}-1)$. Vorticity convection also provides the final, Nth, row of equation 1. Because the wake is modeled with a finite length, the last vortex element must be treated specially. "Otherwise, the starting vortex would disappear abruptly when it reached the end of the computational wake, producing a discontinuous change in the induced wash at the airfoil. To alleviate this difficulty, ... the vorticity is allowed to dissipate smoothly by using a relaxation factor," wrote Hall ${ }^{1}$.

The formulation and analysis of the aerodynamic model progresses in the following manner. Discrete, timemarching equations are written, equation 1 . Once these equations are written, they inherently contain the approximations of the finite wake and the discretization. A discrete Fourier transformation is performed on the unforced equations, producing the z-plane representation, eqn 2 .

$$
z \Gamma_{0}=\left(-A^{-1} B\right)_{0}
$$

The discrete time eigenvalues, $\mathbf{z}$, and the eigenvectors, $\Gamma_{0}$, are extracted from these equations to determine system stability. These eigenvalues are then converted to the continuous time domain, $\lambda$-plane, through a zero order hold transformation, equation 3 .

$$
\lambda=\frac{\log (z)}{\Delta t}
$$

\section{Baseline Configuration}

As the first of several numerical examples, we consider the flow over an airfoil with 20 vortex elements on the airfoil and 180 elements in the wake, equally spaced. This will be referred to as the baseline case. The (finite) length of the wake thus extends 9 chord lengths. The eigenvalues and eigenmodes of the flow can be computed by established methods ${ }^{1}$. Because there are 200 elements in the model, 200 eigenvalues result.

The discrete time (z-plane) eigenvalues, extracted from equation 2, approximately form a circle centered at the origin, as shown in figure 1 . In addition to these eigenvalues, there are a finite number of eigenvalues at the origin. The number of eigenvalues at the origin is equal to the number of segments or grid points on the airfoil. This conclusion follows from examining the rank of the system matrices in equation 1 , from the numerical results obtained here, and appears to be supported by the results presented in reference 1 , though it was not noted in this previous work. Eigenvalues at the origin in the discrete time domain transform to negative infinity in the continuous time domain. 
The continuous time eigenvalue distribution for the baseline case is shown in figure 2 . The real part of the eigenvalue is indicative of the damping and the imaginary part is the damped frequency of each fluid eigenmode. Examining the eigenvalues of the aerodynamic matrix in the continuous domain produces several observations. The continuous domain eigenvalues are discretely spaced and are arranged in "arms" that emanate from the origin and reach up and down in the left half plane. Additionally, the real parts of the arms asymptotically approach a limiting value.

The presence of aerodynamic damping is evidenced by the arms lying in the left half plane. The primary contribution to the damping appears to lie with the overall flow field, however, there is additional damping due to the presence of a vorticity relaxation factor at the last wake element. The relaxation factor used in the vortex lattice model provides energy dissipation in the wake; as the relaxation factor is decreased, more energy is dissipated and the aerodynamic damping increases. If the number of aerodynamic boxes within the wake is increased, the last box will be a smaller percentage of the total wake length and thus, the influence of the relaxation factor will be diminished.

\section{Parametric Variations}

Three aspects of the aerodynamic modeling significantly impact the eigenvalue distribution: the size of the aerodynamic elements, the number of these elements that lie in the wake, and the length of the wake. The three aerodynamic configurations, detailed in table 1, compared against each other two at a time, produce the three comparison cases, which are organized in table 2 and discussed next. The three comparison cases are discussed in terms of their discrete time eigenvalue distributions (z-values), their discrete-to-continuous time domain transformations (z-transformations) and their continuous time eigenvalue distributions ( $\lambda$-values).

Comparison case I compares aerodynamic configurations 2 and 3 , examining the effects of varying the size of the aerodynamic elements while maintaining the number of elements which lie in the wake. Because the number of wake elements remains fixed, configuration \#2 has a wake that is twice the length of the wake in configuration \#3 and elements which are twice as large. Although not shown, the discrete time eigenvalue patterns for configurations 2 and 3 are identical because the number of elements in each wake is identical. However, changing the size of the aerodynamic elements changes the transformation, which must be applied to convert the discrete time system to continuous time. This difference in transformation produces the change in continuous domain eigenvalues, as illustrated in figure 3 .

It is easily shown that the frequency of each eigenvalue scales linearly with the aerodynamic element size. The maximum frequency of the arms can be determined a priori by utilizing Shannon's sampling theorem ${ }^{8}$. The aerodynamic eigenfrequencies are bounded from discrete time considerations similar to those that predetermine the discrete Fourier transform frequencies ${ }^{8.9}$. The maximum frequency, $\omega$, that can be resolved would have 1 cycle spanning two adjacent aerodynamic panels. Using the velocity, $U$, to relate the spatial, $\Delta \mathbf{x}$, and temporal, $\Delta \mathbf{t}$, sample sizes leads to

$$
\max (\omega)=\frac{\pi U}{\Delta x}
$$

Thus, changing the aerodynamic element size changes the frequencies of the aerodynamic eigenvalues. As the size of the elements becomes infinitesimal, we speculate that the eigenvalue arms will cover the frequency range from $+/$ - infinity.

It should be noted in studying Case I that the number of eigenvalues has remained constant in going from configuration 2 to configuration 3 , while the frequency range has doubled. Thus, the density of the eigenvalues has halved. The implications of this will be further discussed in studying Case III.

Comparison case II compares aerodynamic configurations 1 and 2 and examines the effect of varying the number of aerodynamic elements in the wake while holding their size constant. The number of aerodynamic elements in the wake determines the number of discrete time eigenvalues comprising the pseudo-circular pattern. As more elements are placed in the wake, the more crowded pattern expands outward towards the unit circle. As the element size decreases, the radius of the pseudo-circular pattern asymptotically approaches 1 . In discrete time eigenvalue analysis, an eigenvalue lying on the unit circle represents a neutrally stable system. In the continuous time domain, the imaginary axis is the line of demarcation for stability. It is thus anticipated that the additional boxes in the wake force the "arms" of the continuous time eigenvalues closer to the imaginary axis. Figure 4 bears this out. As more elements are added to the wake, the closer the aerodynamic roots get to those associated with simple harmonic motion. Thus, changing the number of aerodynamic elements in the wake changes the damping of the aerodynamic eigenvalues. As the number of elements goes to infinity, we speculate that the arms will move to the imaginary axis. 
It should be noted in studying Case II, as the wake length is increased, leaving the size of the aerodynamic elements constant, the frequency range of the continuous time eigenvalues remains constant. Doubling the number of elements in the doubles the number of eigenvalues on the "arms." Twice as many eigenvalues reside in arms of the same length. Hence, the continuous time eigenvalue distribution has become denser.

Comparison case III compares aerodynamic configurations 1 and 3 and examines the effects of varying simultaneously and in inverse proportion, the number and length of aerodynamic elements in the wake, such that the wake length remains constant. The expected trends for the behavior of the arms of the continuous time eigenvalues are difficult to predict because, in going from configuration 1 to configuration 3 there are multiple tendencies: increasing the number of elements tends to move the arms closer to the imaginary axis; decreasing element size tends to extend the frequency range of the arms. The combined result on the continuous time eigenvalues, shown in Figure 5, is that the arms of the eigenvalues lie approximately the same distance from the imaginary axis, while the frequency range of configuration 3 is twice that of configuration 1. This corresponds to the effects of smaller element size of configuration 3 . Thus, the spacing of the eigenvalues is approximately constant between the two analysis runs.

An approximate formula for eigenvalue spacing is derived using the frequency range and the number of eigenvalues. The maximum frequency was found using equation 4. Accounting for positive and negative values, the frequency range is twice this. Dividing this range by the number of elements or eigenvalues in the wake, and recognizing that the element size times the number of elements in the wake is the wake length, $\mathbf{L}_{\text {wake, }}$ produces the relationship

$$
\Delta \omega=\frac{2 \pi U}{L_{\text {wake }}} \quad(\text { Eqn.5) }
$$

The reader may recognize that this is similar to determination of the discrete Fourier transformation frequencies, as determined by the length of the time record. The eigenvalue spacing is approximate due to the eigenvalues not lying on the imaginary axis, that is, due to the discretization-induced damping. For the case of the element size becoming infinitesimally small, the formula is exact.
Thus, the effect of the finite wake is to produce discretely spaced eigenvalues, instead of a continuous line. As the wake length becomes infinite, we speculate that the arms of discretely spaced eigenvalues form continuous lines emanating from the origin.

\section{$\underline{\text { Discussion }}$}

The study of aerodynamic eigenvalues using the vortex lattice code has led to some basic ideas. The eigenvalues have been shown to be artifacts of the discretization and the finite length wake.

The effects of discretization are controlled by two independent factors. The size of the elements determines the range of frequencies covered by the eigenvalues, while the number of elements in the wake drives the damping. Their effects are shown to be independent, as one controls the transformation from discrete to continuous time, and the other controls the discrete time eigenvalue pattern. The effect of the finite wake is to produce discretely spaced eigenvalues, instead of a continuous line.

We offer the following speculations regarding the limiting cases. As the size of the elements becomes infinitesimal, the eigenvalue arms will cover the frequency range from $+/$ - infinity. As the number of elements goes to infinity, the arms will move to the imaginary axis. As the wake length becomes infinite, the arms of discretely spaced eigenvalues form continuous lines emanating from the origin.

\section{AEROELASTIC STUDIES}

With the ability to model aerodynamic eigenmodes as well as structural modes, we now have the capability to investigate the coupled fluid/structural modes or aeroelastic modes. We study these in this section of the paper and thereby gain additional insight into the behavior of such system. One intriguing finding, previously foreshadowed in the literature, is that the critical mode may originate in an aerodynamic mode rather than a structural mode.

\section{Aeroelastic Modeling}

The discrete time aerodynamic model can be coupled with a discretized structural dynamic model to produce the following time-marching aeroelastic equations of motion ' which can then be analyzed to determine the behavior of the system

$$
D_{2} q^{n+1}+D_{1} q^{n}-f^{n+1}=0
$$

where the vector $q$ contains the structural dynamic degrees of freedom, the vector $f$ represents the 
aerodynamic loads and the matrices $D_{1}$ and $D_{2}$ describe the structural dynamic behavior of the typical section.

The coupling between the aerodynamic and the structural dynamic quantities is present in an aeroelastic system because the vorticity distribution results in the aerodynamic loads, $f$, which can be expressed in terms of the vorticities, $\Gamma$.

$$
f^{n+1}=C_{2} \Gamma^{n+1}+C_{1} \Gamma^{n}
$$

For an unforced response, the downwash on the airfoil, w, is produced by the motion of the airfoil.

$$
w^{n}=E q^{n}
$$

Combining equations $1,6,7$, and 8 produces the unforced aeroelastic system equations.

$$
\left[\begin{array}{cc}
D_{2} & C_{2} \\
-E & A
\end{array}\right]\left\{\begin{array}{l}
q \\
\Gamma
\end{array}\right\}^{n+1}+\left[\begin{array}{cc}
D_{1} & C_{1} \\
0 & B
\end{array}\right]\left\{\begin{array}{l}
q \\
\Gamma
\end{array}\right\}^{n}=\left\{\begin{array}{l}
0 \\
0
\end{array}\right\}
$$

\section{Aeroelastic Configuration}

A typical section with only a single pitch degree of freedom, figure 6, is analyzed in this paper. For these analyses, 10 aerodynamic elements were placed on the wing; 90 aerodynamic elements were placed in the wake. The structural parameters are given in Table 3.

The stability of this system was analyzed by calculating the equations of motion for a series of reduced velocities. Reduced velocity, $\mathrm{V}$, is defined as the velocity, $U$, normalized by the pitch frequency and semi-chord. Eigenanalyses of the discrete time systems were performed on each set of equations and the system eigenvalues tracked. The eigenvalues were transformed into the continuous time domain using a zero order hold transformation. Stability can be inferred from either the discrete or the continuous time root locus- both are examined here.

Discrete Time Eigenvalues - The discrete time root locus is presented in figure 7; only the portion of the complex plane near the unit circle's intersection with the real axis is shown. The coupled pitch mode-originating eigenvalue and the aerodynamic-originating eigenvalues, referred to as the aeroelastic eigenvalues, migrate as the reduced velocity is increased. The single structural dynamic eigenvalue can be seen near the unit circle, indicating that it is more lightly damped than the aerodynamic eigenvalues.
An instability occurs when an eigenvalue lies outside the unit circle. For this system, the instability is observed by examining the real aerodynamic-originating eigenvalues on the positive real axis. Figure 7 shows the migration of the structural-dynamic-originating eigenvalue, and also the interplay with several aerodynamic eigenvalues. The lowest complex aerodynamic eigenvalue is clearly influenced, as well as the real aerodynamic eigenvalues, one of which destabilizes. It is difficult to further study system behavior from these graphs because each velocity produces eigenvalues that essentially belong in different z-planes. This will be discussed in detail in a subsequent section of this paper. For a more intuitive interpretation, we turn now to the continuous domain eigenvalues for this aerodynamic model.

Continuous Time Eigenvalues -...The systems are converted to the continuous domain by zero order hold transformations. The behavior of the continuous time domain eigenvalues is shown in figure 8 . For clarity, only the region near the origin is presented. The influence of velocity on the aerodynamic eigenvalues is now evident. As velocity increases, the eigenvalues' frequencies increase at constant damping. This particular configuration destabilizes at zero frequency, termed divergence. In figure 8 , the eigenvalues at the divergence velocity, $V=2.3$, are indicated by square symbols.

It is interesting to note that the eigenvalue of the pitch mode does not go to zero as divergence occurs. The pitch mode eigenvalue maintains a non-zero frequency as the system destabilizes, as one of the real aerodynamic roots migrates into the right half plane. This is contrary to conventional wisdom regarding divergence, although several similar phenomena have been reported by Dashcund ${ }^{10}$, Edwards ", Rodden and Stahl ${ }^{12}$, and Rodden and Bellinger ${ }^{13}$. The mechanism responsible for the divergence of this configuration appears to have its origin in the aerodynamic terms. Further work is planned to determine the physical and mathematical significance of this discovery.

Modal Participation - Often neglected when interpreting the results of an eigenanalysis, the eigenvectors provide much interesting information. Afolabi, Pidaparti and Yang ${ }^{14}$ studied aeroelastic stability from the standpoint of system eigenvectors. In their work, they discuss the orthogonality between eigenvectors associated with the different modes of the system. The approach taken in the current work is to examine the eigenvectors associated with individual modes. The eigenvector associated with a particular eigenvalue can be viewed as the set of modal participation factors for each degree of freedom. Note 
that the eigenvectors are invariant under the transformation from discrete to continuous time domain.

In a numerically stiff set of ordinary differential equations ${ }^{15}$, the system behavior is seen to be dominated by the lightly damped and unstable modes. The disparity in the time scales of components of the system allows the overall behavior to be studied by observing only a few eigenmodes of the system. Thus, in studying the system behavior, only the modal participation factors associated with the least stable dynamic mode and with the least stable static mode were examined. Here, the complex mode corresponds to the one that originated as the structural pitch mode, and the real mode corresponds to an eigenvalue that originated in the aerodynamics.

Vorticity Associated with the Pitch ModeThe modal participation at a low reduced velocity, $V=0.2$, is presented for the mode which is primarily the pitch degree of freedom, figure 9 . The corresponding eigenvalue is identified in figure 8 by the diamond symbol. For clarity, only the real part of the modal participation is plotted as a function of chord-wise or downstream position. At this low velocity, the aerodynamics are being driven at the frequency of the structural mode. The portion of the eigenvector associated with the vorticity at each aerodynamic control point, referred to as the vorticity participation, shows that most of the aerodynamic energy associated with this mode is in the wake. The first ten participation factors correspond to elements on the airfoil. Only these vorticities can produce forces on the airfoil. At this velocity, there is very little aerodynamic energy being imparted to the airfoil.

The wake portion of the vorticity participation appears as a negatively damped sinusoid when viewed spatially, as in figure 9 . The eigenvector provides a snapshot of the vorticity distribution. Initial examination of the data in figure 9 may lead one to conclude that the system is unstable. In fact, the opposite is indicated. For a stable system, the vorticity being shed from the wing into the wake will decrease as time advances. The vorticity on the last wake element at time $\mathrm{n}$ is the same as the vorticity on the first wake element at time $n-\mathrm{N}_{\text {wake. Thus, the }}$ spatial vorticity distribution could also be thought of as a time history, where time originates at the wake trailing edge and proceeds towards the airfoil.

Near the divergence reduced velocity, the eigenvector associated with the structural-dynamic-originating mode contains significant participation from both the structural dynamic and the aerodynamic states. Figure 10 shows the vorticity participation spatially for a velocity just below divergence, $V=2.3$. The number of oscillations to be expected in the wake, $\mathbf{N}_{\text {cycles, }}$ can be estimated using the frequency of the associated eigenvalue, $\omega_{\text {mode, }}$ the reduced velocity, $\mathbf{V}$, and the discretization, $\mathbf{N}_{\text {wake }}$ and $\mathbf{M}$ :

$$
N_{\text {cycles }}=\frac{\omega_{\text {mode }} N_{\text {wake }}}{\omega_{\alpha} M \pi V}
$$

Using the values for the divergence condition results in a prediction of 0.8 oscillations; the vorticity participation in figure 10 therefore looks reasonable.

Vorticity Associated with the Real Aerodynamic ModeThe aeroelastic system studied destabilizes as a real eigenvalue moves into the right half plane. It is interesting to examine the progression of the characteristics of this mode as the reduced velocity increases. Regardless of reduced velocity, the associated vorticity participation factor resembles a pressure coefficient distribution on the airfoil elements, while the wake contains almost no participation except for the last element. The vorticity participation factor at an example reduced velocity, $V=2.3$, is presented in figure 11 . As the reduced velocity changes, it is the participation of the last wake element which is interesting. The magnitude of this term is plotted versus reduced velocity in figure 12. As this plot is examined, note that these eigenvectors have an overall magnitude of 1 . Initially, nearly all of the vorticity participation resides in the last element of the wake. Just prior to divergence, the participation drops sharply. At the divergence velocity, all of the vorticity participation is on the airfoil; the wake factors are zero. As the system moves beyond the divergence velocity, the behavior of all of the vorticity participation factors change. The last wake element quickly becomes influential again, but now with vorticity that is negative, or out of phase, with the airfoil vorticity. As velocity is further increased, the participation of the last wake element smoothly, asymptotically, approaches zero. Also beyond divergence the overall wake vorticity participates.

Transition from stability to instability produces dramatic changes in the associated eigenvector. While the eigenvalue smoothly traverses across the imaginary axis, the character of the vorticity participation changes sharply. Future work will focus on understanding the nature of this instability through examination of the eigenvectors. 
Computational Issues for Simultaneous Solution of Aerodynamic and Structural Equations

Transformation Compatibility - To incorporate the discrete time aerodynamic model into aeroelastic equations, the structural dynamic model must be cast in discrete time also. The structural dynamic equations contain first and second derivatives that could be approximated using a central difference technique. While this is convenient and easy, this method results in a mismatch of discrete time transformations. Central differencing produces discrete time equations to which a first order Tustin transformation ${ }^{9}$

$$
\lambda=\frac{2}{\Delta t} \frac{(z-1)}{(z+1)} \quad(\text { Eqn.11) }
$$

must be applied to obtain the proper continuous time results. The Tustin transformation is equivalent to the first term in a series expansion of the zero order hold transformation presented in equation 3 . In these transformations, the sample interval, $\Delta \mathbf{t}$, establishes the relationship between the discrete time eigenvalues, $\mathbf{z}$, and the continuous time eigenvalues, $\lambda$. The aerodynamic equations which were generated with a zero order hold discretization, are solved simultaneously with the discretized structural dynamic equations. Thus, it is desirable to have structural dynamic equations that would also be correct when a zero order hold transformation is applied. This is easily accomplished through standard discretization techniques ${ }^{9}$. Accepting the mismatch in the transformations results in a phenomenon that resembles aliasing. However, as the time step becomes small, the zero order hold transform and the Tustin transform become approximately equivalent.

Aliasing - The equations have been constructed in the discrete time domain. Given data at discrete times, we can utilize a transformation to approximate the response in continuous time. There are limitations to discrete time transformation methods; aliasing is the primary concern ${ }^{8,9}$. To avoid aliasing, a continuous time signal must have 2 samples per period of period of the highest frequency to be resolved. The aerodynamic equations arose from the fundamental concept of vorticity being convected downstream at a velocity, $U$. The equations are valid only if the relationship $U=\Delta x / \Delta t$ is maintained. It is thus observed that the minimum velocity, at which the system may be accurately analyzed, is set by the spatial discretization and the maximum frequency that is important to the problem. Another interpretation is that for frequency and velocity ranges of interest, the minimum number of aerodynamic elements required to avoid aliasing can be approximated. This can serve as a guideline in selecting the spatial discretization required for a given problem. There are additional implications of the discrete time effects when the aerodynamic equations are combined with the structural dynamic equations or control laws.

Methods of Stability Analysis - The aeroelastic stability analyses, which require variation of the velocity, were performed using a single spatial aerodynamic discretization. This was accomplished $b$ adjusting the temporal discretization to produce the proper velocities. There are several complications in performing the analyses in this manner: (1) a separate transformation rule must be applied for each velocity; and (2) interpreting the discrete time eigenvalues is not intuitive. The aerodynamic matrices were unchanging for different velocities, but the matrices which couple them to the structural dynamics were not. The resulting aeroelastic eigenvalues change with each velocity. The migration of the eigenvalues in the discrete time domain is not due solely to the velocity change, but to a combination of velocity and sample rate change.

A brief study was conducted to look at the results when a consistent sample rate was utilized, meaning that as the velocity changed, the spatial discretization changed. This required constructing a new aerodynamic model at each velocity. There was negligible effect on the continuous time eigenvalues. The discrete time eigenvalue pattern associated with the structural dynamic mode changed significantly. It was observed, however, that the discrete time eigenvalue pattern in this case is nearly identical to the pattern produced when the eigenvalues from the nominal analysis method are rediscretized using the consistent sample rate.

\section{CONCLUDING REMARKS}

Aerodynamic and aeroelastic eigenanalyses were performed utilizing a time-domain vortex lattice aerodynamic code, coupled with discretized structural dynamic equations. The study of aerodynamic eigenvalues using the vortex lattice code has led to some basic ideas. The eigenvalues have been shown to be artifacts of the discretization and the finite length wake. The effects of discretization are controlled by two independent factors. The number of elements in the wake drives the damping, while the size of the elements determines the range of frequencies covered by the eigenvalues. The effect of the finite wake is to produce discretely spaced eigenvalues, instead of a continuous line. 
Aeroelastic analyses were performed to examine the stability and modal character as a function of reduced velocity. Insight into the modeling and physics associated with system behavior can be gained by examining the eigenvectors. A novel determination is that under some circumstances an eigenmode of aerodynamic origin can be the critical mode for aeroelastic instability.

\section{REFERENCES}

1 Hall, K.C, Eigenanalysis of unsteady flows about airfoils, cascades and wings, AIAA Journal Vol. 32, No. 12, December 1994

2 Dowell, E.H., Hall, K.C, and Romanowski, M.C., Eigenmode analysis in unsteady aerodynamics: reduced order models, Applied Mechanics Review Vol. 50, \#6, p371-385, June 1997.

3 Hassig, H.J., An approximate true damping solution of the flutter equation by determinant iteration, Journal of Aircraft Vol. 8, No. 11, November 1971.

4 Rodden, W.P., and Bellinger, E.D, Aerodynamic Lag Functions, Divergence, and the British Flutter Method, Journal of Aircraft, Volume 19, Number 7, July 1982.

5 Rodden, W.P., Harder, R.L., and Bellinger, E.D, Aeroelastic Addition to NASTRAN, NASA Contractor Report 3094, 1979.

6 Bisplinghoff, R.E., Ashley, H., and Halfman, R.L., Aeroelasticity, Dover Publications, Mineola, New York, 1996 (copyright Addison-Wesley Publishing Company, 1955).

7 Dowell, E.H., Crawley, E.F., Curtis, H.C.,Jr., Peters, D.A., Scanlan, R.H., and Sisto, F., A modern course in aeroelasticity, Kluwar Academic Publishers, Dordrech, The Netherlands, $1995,3^{\text {rd }}$ edition.

8 Hardin, J.C., Introduction to Time Series Analysis, NASA Reference Publication 1145, p83-88, Nov 1990.

9 Oppenheim, A.V., and Shafer, R.W., Discrete time signal processing, Prentice Hall, Englewood Cliffs, New Jersey, 1989.

10 Dashcund, D.E., The development of a theoretical and experimental model for the study of active suppression of wing flutter, MAE Technical Report 1496T, Princeton University, December 1980.

11 Edwards, J.W., Unsteady aerodynamic modeling and active aeroelastic control, SUDAAR 504, Department of Aeronautics and Astronautics, Stanford University, February 1977.
Rodden, W.P., and Stahl, B., A Strip Method for Prediction of Damping in Subsonic Wind Tunnel and Flight Flutter Tests, Journal of Aircraft, Vol 6, No. 1 (Jan-Feb 1969).

13 Rodden, W.P., and Bellinger, E.D, Unrestrained Aeroelastic divergence in a Dynamic Stability Analysis, Journal of Aircraft, Volume 19, Number 9, September 1982

14 Afolabi, D, Pidaparti, R.M.V., and Yank, H.T.Y, Flutter Prediction Using an Eigenvector Orientation Approach, AIAA Journal Vol 36, No 1, January 1998.

15 Kincaid, D.R. and Cheney, E.W., Numerical analysis: Mathematics of scientific computing, p 556 Brooks/Cole Publishing Company, Pacific Grove, California, 1991. 


\begin{tabular}{|c|c|c|c|c|c|c|}
\hline \multirow{2}{*}{$\begin{array}{c}\text { Aero Config } \\
\text { No. }\end{array}$} & \multicolumn{3}{|c|}{ Airfoil } & \multicolumn{3}{|c|}{ Wake } \\
\hline & $\begin{array}{c}\text { No. of } \\
\text { elements }\end{array}$ & $\begin{array}{l}\text { Normalized } \\
\text { element size }\end{array}$ & $\begin{array}{l}\text { Normalized } \\
\text { airfoil length }\end{array}$ & $\begin{array}{c}\text { No. of } \\
\text { elements }\end{array}$ & $\begin{array}{l}\text { Normalized } \\
\text { element size }\end{array}$ & $\begin{array}{c}\text { Normlized } \\
\text { wake length }\end{array}$ \\
\hline $\begin{array}{c}1 \\
\text { (Baseline) }\end{array}$ & 20 & 1 & 1 & 180 & I & 1 \\
\hline 2 & 20 & 1 & 1 & 360 & 1 & 2 \\
\hline 3 & 40 & $1 / 2$ & 1 & 360 & $1 / 2$ & 1 \\
\hline
\end{tabular}

Table 1. Aerodynamic Configurations

\begin{tabular}{|c|c|c|c|c|c|}
\hline \multirow[t]{2}{*}{$\begin{array}{l}\text { Comparison } \\
\text { Case No. }\end{array}$} & \multicolumn{3}{|c|}{$\begin{array}{c}\text { Aerodynamic Configurations } \\
\text { Compared }\end{array}$} & \multirow[t]{2}{*}{ Parametric Variation } & \multirow[t]{2}{*}{ Quantity Held Constant } \\
\hline & 1 & 2 & 3 & & \\
\hline I & & $\checkmark$ & $\checkmark$ & $\begin{array}{l}\text { Size of aerodynamic } \\
\text { elements in wake }\end{array}$ & $\begin{array}{c}\text { Number of aerodynamic } \\
\text { elements in wake }\end{array}$ \\
\hline II & $\checkmark$ & $\checkmark$ & & $\begin{array}{c}\text { Number of aerodynamic } \\
\text { elements in wake }\end{array}$ & $\begin{array}{l}\text { Size of aerodynamic } \\
\text { elements in wake }\end{array}$ \\
\hline III & $\checkmark$ & & $\checkmark$ & $\begin{array}{l}\text { Size and number of } \\
\text { elements in wake }\end{array}$ & Length of wake \\
\hline
\end{tabular}

Table 2. Comparison Cases for Parametric Variations

\begin{tabular}{|l|l|}
\hline Semi-chord, $(\mathrm{b})$ & 2.875 inches \\
\hline $\begin{array}{l}\text { Distance from midchord to elastic axis, } \\
\text { Non-dimensionalized by semi-chord, }(\mathrm{a})\end{array}$ & -0.1304 \\
\hline Span, $(\mathrm{S})$ & 4 inches \\
\hline Radius of Gyration, $\left(\mathrm{r}_{\alpha}\right)$ & 0.41 \\
\hline Mass ratio, $(\mu)$ & 23.1 \\
\hline Pitch frequency, $\left(\omega_{\alpha}\right)$ & 50.2 radians/second \\
\hline $\begin{array}{l}\text { Distance from aerodynamic center to elastic axis, } \\
\text { non-dimensionalized, }(\mathrm{e} / \mathrm{b})\end{array}$ & 0.37 \\
\hline
\end{tabular}

Table 3. Structural Parameters of Typical Section 




Figure 1 Eigenvalues for baseline case Discrete time eigenvalues, $\mathrm{z}$



Figure 3 Case I: Influence of varying the size of the aerodynamic elements. Continuous time eignvalues, 1

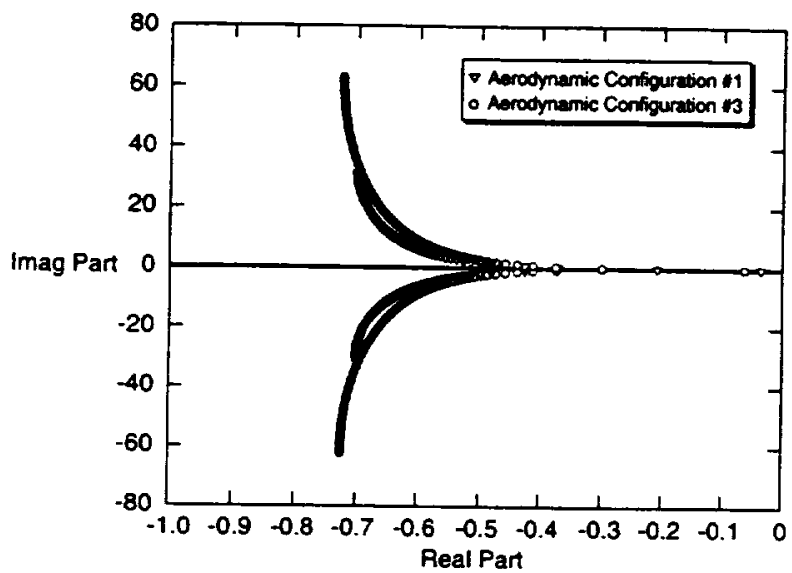

Figure 5 Case III: Influence of simultaneously varying the size and number of aerodynamic elements in the wake, maintaining a constant wake length.

Continuous time eignvalues, 1

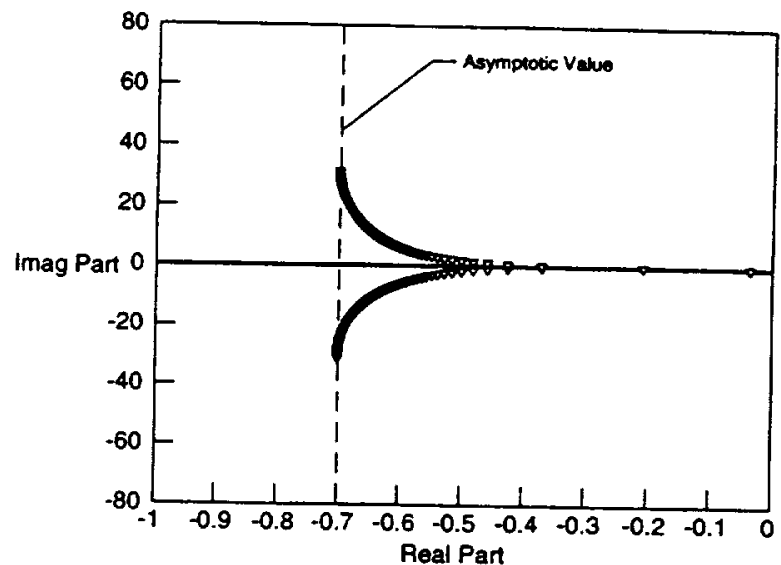

Figure 2 Eigenvalues for baseline case Continuous time time eigenvalues, 1

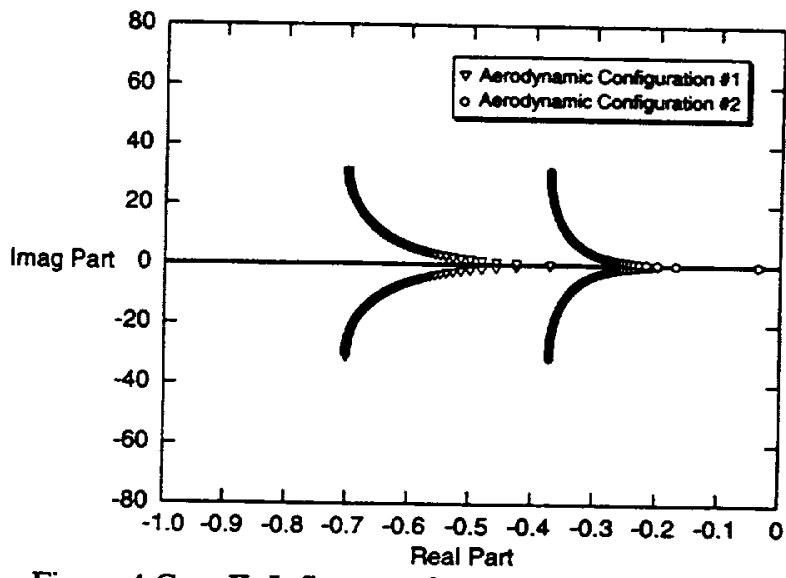

Figure 4 Case II: Influence of varying the number of aerodynamic elements in the wake. Continuous time eigenvalues, 1

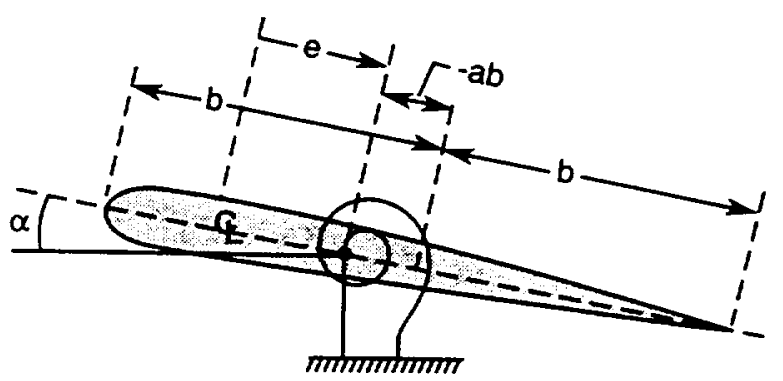

Figure 6 Typical section with pitch freedom. 


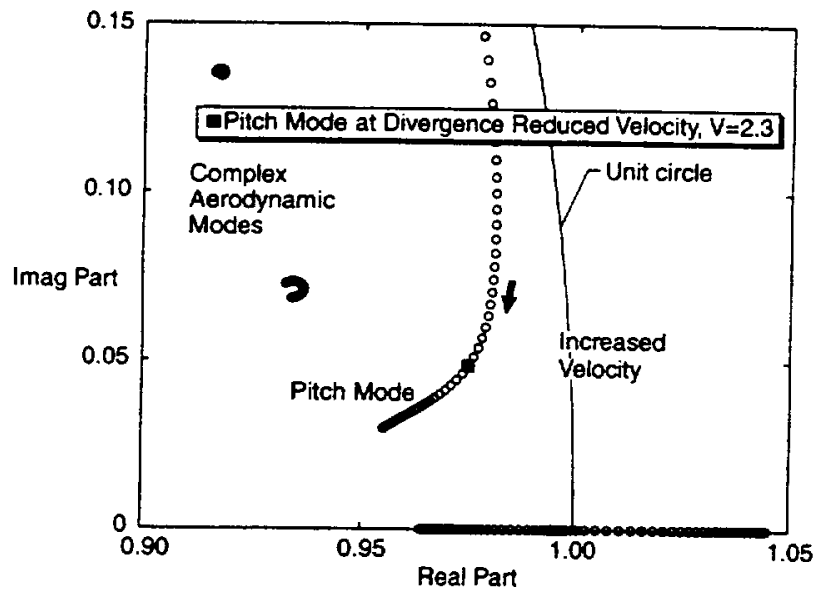

Figure 7 Discrete Time Aeroelastic Eigenvalues as Reduced Velocity is Varied

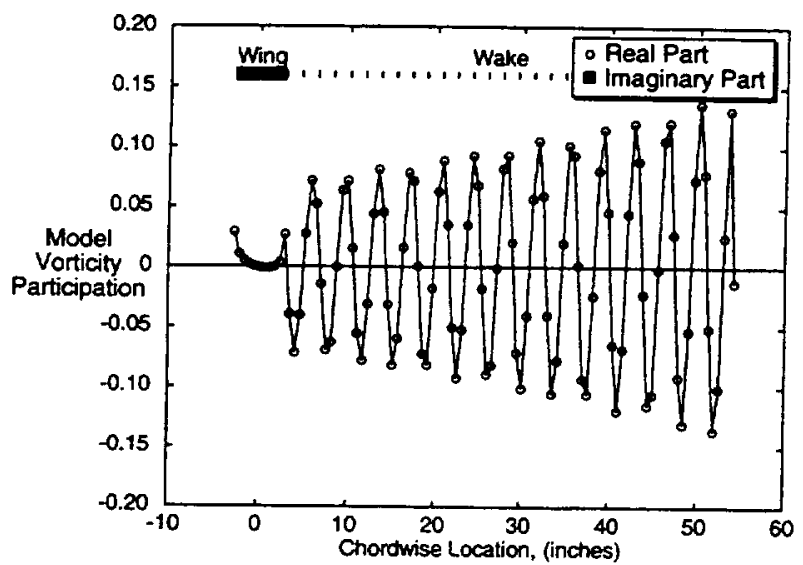

Figure $9 \mathrm{~V}=0.2$, Eigenvector associated with pitch originated mode, $\lambda=0.40354+j 49.274$

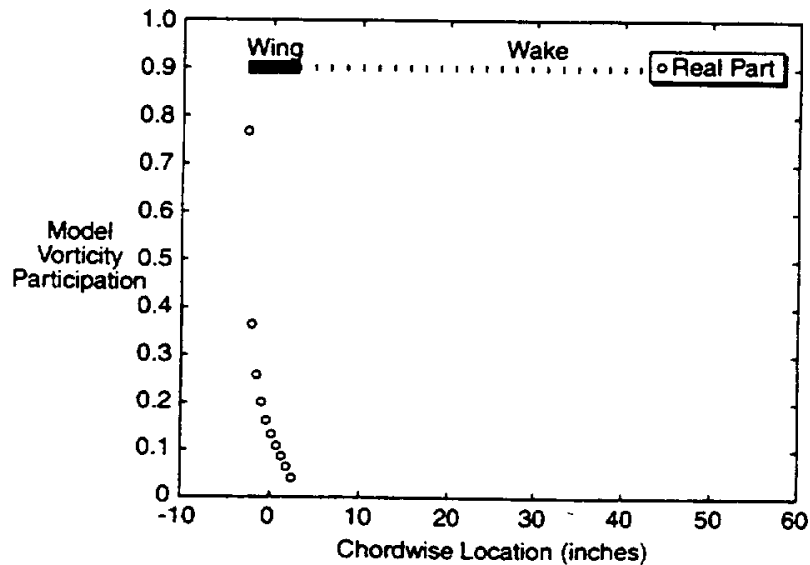

Figure $11 \mathrm{~V}=2.3$, Eigenvector associated with real aerodynamic modes, $\lambda=0.43435+\mathrm{jC}$



Figure 8 Continuous Time Aeroelastic Eigenvalues as Reduced Velocity is Varied

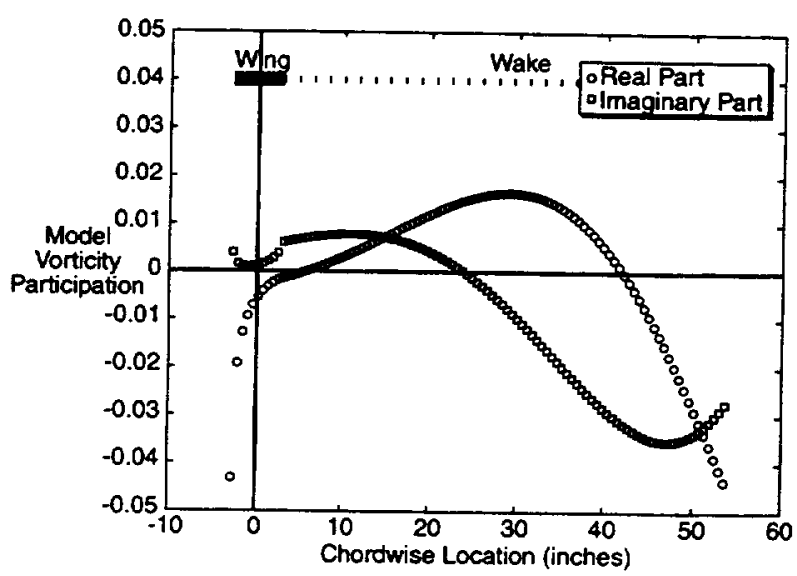

Figure $10 \mathrm{~V}=2.3$, Eigenvector associated with pitch originated mode, $\lambda=13.9111+j 28.7958$



Figure 12 Vorticity participation of last wake element for real eigenvalue 
